\title{
ON AMATI RELATION FOR GRB PROMPT EMISSION
}

\author{
L. Titarchuk ${ }^{1}$ and R. Farinelli ${ }^{1}$
}

\begin{abstract}
We propose a model for the spectral formation of Gamma Ray Burst (GRB) prompt emission, where the phenomenological Band function is usually applied to describe the GRB prompt emission. We suggest that the GRB prompt emission is mainly a result of the Comptonization of relatively soft photons of the star off electrons of a hot shell of plasma of temperature of the order of $10^{9} \mathrm{~K}$ ( or $\left.\sim 100 \mathrm{keV}\right)$ that moves sub-relativistically with the bulk velocity substantially less than the speed of light. In this case, the Comptonization parameter is high and the interaction between a blackbody-like soft seed photon population and hot electrons leads to formation of a saturated Comptonization spectrum. We give an interpretation of the Amati relation between the intrinsic spectral peak photon energy and radiated energy or luminosity.
\end{abstract}

\section{Introduction}

Understanding the physical processes which give rise to the observed spectra of the prompt emission of Gamma Ray Bursts (GRB) is presently one of the most exciting issues studied by both the theoretical and observational community. The Band function (Band et al. 1993) up to now widely used to describe their prompt emission is a pure phenomenological model. It consists of two low-energy and highenergy power laws with photon index $\Gamma_{1}$ and $\Gamma_{2}$, respectively, smoothly joined at some energy $E_{\mathrm{b}}$. We offer a model to explain the GRB prompt spectral formation in the context of a photospheric scenario in which the main process is the Comptonization of the relatively soft photons of the star by a hot sub-relativistic outflow of velocity $V_{b}$ within an area close to the photospheric radius (optical depth of 3-5) likely symmetric with respect to the rotational axis of the star (see Fig. 1 for illustration of our model). We use the theoretical and numerical results of the Comptonization problem reported in Titarchuk et al. (1997) and Farinelli et al. (2008) and finally in Titarchuk et al. (2012, hereafter TFFA12) for the case of

${ }^{1}$ Dipartimento di Fisica, Università di Ferrara, via Saragat 1, 44122 Ferrara, Italy 

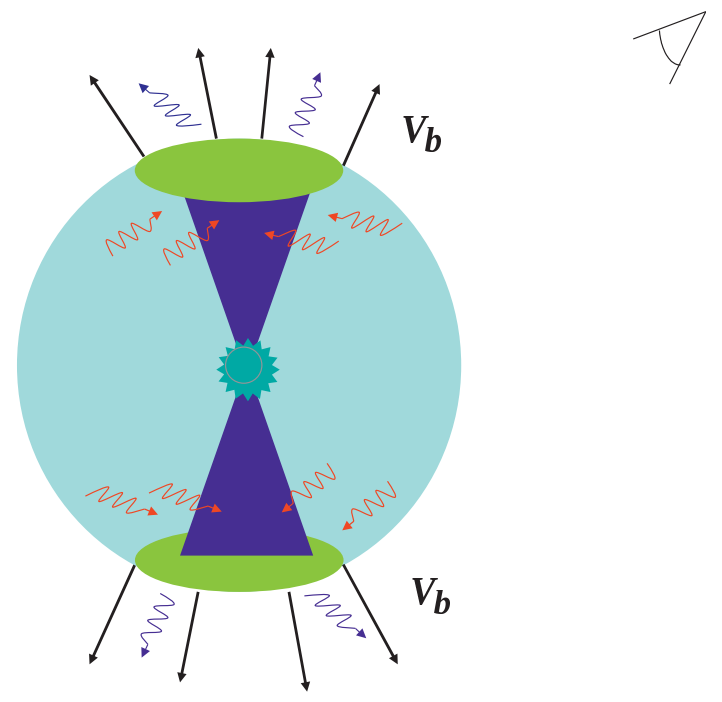

Fig. 1. X-ray spectral formation during explosion of a massive star (see also TFFA12).

an early subrelativistic bulk outflow phase produced during the supernova explosion. In Section 2 we demonstrate that our upscattering model of GRB radiation reproduces the Amati relation between the $E F(E)$ peak energy $E_{\mathrm{p}}$ and the GRB radiated energy $E_{i s o}$ or luminosity $L_{g r b}$. In Section 3 we draw our conclusions.

\section{The Amati relation and its interpretation}

In spite of the still open discussion about the impact of possible selection effects on the correlation between the peak energy of $E F(E)$ diagram $E_{p}$ and the GRB radiated energy or luminosity (Amati relation, see Amati et al. 2002) it is a matter of fact that all GRBs with known redshifts, but one (GRB 980425), follow this relation (Amati et al. 2009). Thus it is crucial to understand the origin of this relation, namely which is the mechanism (at first glance universal) which gives rise to the relation $E_{\mathrm{p}} \propto E_{\text {iso }}^{1 / 2}$ where $E_{\mathrm{p}}$ and $E_{\text {iso }}$ are the peak energy of $E F(E)$ diagram and isotropic radiated energy (fluence) of GRB respectively during the prompt phase.

The main issue to be investigated is to check how the parameters of our model concur in determining the energy peak value $E_{\mathrm{p}}$ in the $E F(E)$ diagram and the total luminosity $L_{\text {grb }}$ which integral over prompt emission time is $E_{\text {iso }}$. In Figure 2 we show the resulting Comptonization spectra which also include effect of the early sub-relativistic phase. The spectra becomes softer when the bulk parameter $\delta$ increases from 0.5 to 0.95 (see TFFA12 for details). We should emphasize that $E_{\mathrm{p}}$ is independent of values of the BB-like seed photon temperature $k T_{\mathrm{bb}}$. Thus 


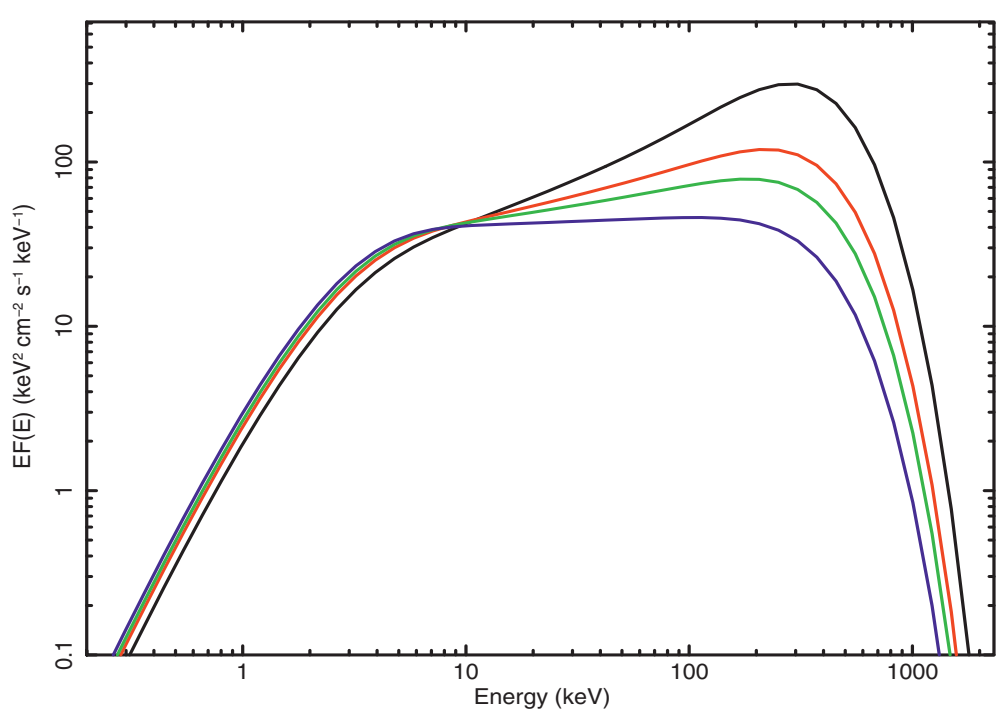

Fig. 2. Saturated Comptonization spectra modified by subrelativistic outflow (see TFFA12).

different values of $k T_{\mathrm{bb}}$ do not change $E_{\mathrm{p}}$, but they determine the total (resulting) luminosity, as we discuss below.

The question that naturally arises is whether the observed dependence of $E_{\mathrm{p}}$ on $E_{\text {iso }}$ (or on luminosity $L_{\text {grb }}$ ) and its intrinsic dispersion (Amati et al. 2008) is the fundamental effect of $\gamma$ - ray emission.

As an example, in Figure 3 we report the results of the fit using our model (see TFFA12) of the time averaged prompt emission spectrum of GRB 990705 obtained with BeppoSAX.

The values of the best fit parameters of our model correspond to the case of the saturated Comptonization for which the resulting spectral index $\alpha \ll 1$ (see details in TFFA12). Sunyaev \& Titarchuk 1980, 1985 derive the formula for the Comptonization enhancement factor $\eta_{\text {comp }}$ which is a ratio of the resulting luminosity, that is in our case, the GRB luminosity $L_{\text {grb }}$ to the injected luminosity of soft photons $L_{\text {soft }}$.

Namely

$$
\eta_{\mathrm{comp}}=\frac{L_{\mathrm{grb}}}{L_{\mathrm{soft}}}=q_{x_{0}}(\alpha) x_{0}^{\alpha-1}
$$

where

$$
q_{x_{0}}(\alpha)=\frac{\alpha(\alpha+3) \Gamma(\alpha+4) \Gamma(\alpha) \Gamma(1-\alpha)}{\Gamma(2 \alpha+4)}\left(1-x_{0}^{1-\alpha}\right),
$$

$x_{0}=2.7 k T_{\mathrm{bb}} / k T_{\mathrm{e}}, k T_{e}$ is the electron temperature of the hot Compton spot (see Fig. 1) and $\Gamma(x)$ is the Gamma function. Thus when $\alpha \ll 1$

$$
\eta_{\text {comp }} \propto k T_{\mathrm{e}}
$$




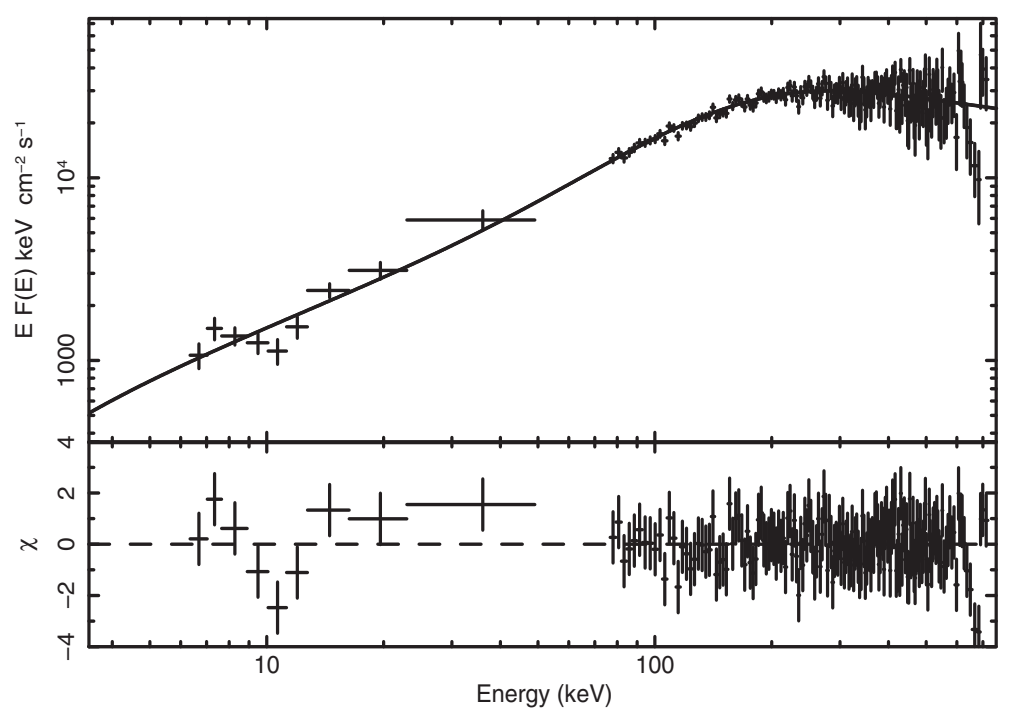

Fig. 3. The best-fit of EF(E) BeppoSAX/WFC+GRBM diagram of GRB 990705 (see TFFA12).

But the flux of soft photons illuminating the hot spot (Compton cloud) $L_{\text {soft }}$ is

$$
L_{\mathrm{soft}}=\pi B_{\mathrm{soft}} S_{\mathrm{hs}}
$$

where $B_{\text {soft }}$ is the intensity of the blackbody radiation of the star and $S_{\mathrm{hs}}$ is the surface area of Compton hot spot (see Fig. 1).

The thermal wave propagates in the hot spot with plasma velocity $V_{p}$ whose value can change from one GRB to another.

As a consequence for each GRB $S_{\mathrm{hs}} \propto\left(V_{p} t\right)^{2}$ (see Fig. 1) and then

$$
S_{\mathrm{hs}} \propto V_{p}^{2} \propto k T_{\mathrm{p}}=k T_{\mathrm{e}} .
$$

Thus the luminosity of the GRB hot spot $L_{\text {grb }}$ should be

$$
L_{\text {grb }}=\eta_{\text {comp }} L_{\text {soft }} \propto\left(k T_{\mathrm{e}}\right)^{2} .
$$

In order to calculate the GRB fluence $E_{\text {iso,grb }}$ one should integrate $L_{\text {grb }}$ over the GRB prompt emission time $T_{p r}$, namely

$$
E_{\mathrm{iso}}=\int_{0}^{T_{p r}} L_{\mathrm{grb}}(t) d t .
$$

If the time-scale of the GRB prompt emission and its shape is more less the same for any burst then $E_{\mathrm{p}}\left(\propto k T_{\mathrm{e}}\right)$ is $E_{\text {iso }}^{1 / 2}$ which is precisely seen in the Amati relation (see Amati et al. 2002). 


\section{Discussion and conclusion}

We have developed a spectral model aimed to describe the broadband prompt emission of GRBs. We propose that the spectral emission during the prompt phase, phenomenologically modeled by the Band function, is the result of an earlier phase where soft BB-like photons are Comptonized by an optically thick and hot electron shell $\left(T_{\mathrm{e}} \sim 10^{9} \mathrm{~K}\right)$, something like a Compton cloud sub-relativistically moving outwards the star surface. On the other hand in the relativistic phase, these Comptonized photons are subjected to a second upscattering process which can be mathematically described by a broken powerlaw Green function whose spectral index models the high-energy slope of the Band function. An important prediction of our proposed model is that the peak energy in the $\mathrm{EF}(\mathrm{E})$ diagram originated in the early subrelativistic phase (see Fig. 1) is directly related to the plasma temperature of the hot plasma $T_{e}$. We demonstrate that the resulting luminosity of $X / \gamma$-rays luminosity of $G R B L_{\mathrm{grb}}$ is proportional to $\left(k T_{\mathrm{e}}\right)^{2}$ (see Eq. (2.6)).

In fact, $L_{\text {grb }}$ is a product of the Comptonization enhancement factor $\eta_{\text {comp }}$ and luminosity of the soft blackbody photons $L_{b b, i l}$ but in the case of the saturated Comptonization, when $\alpha \ll 1, \eta_{\text {comp }} \propto k T_{\mathrm{e}}$ (see Eq. (2.1)) but $L_{\mathrm{bb}, \mathrm{il}}$ (or $L_{\mathrm{soft}}$ ) is also proportional to $k T_{\mathrm{e}}$ because the surface area of Compton cloud illuminated by the soft photons is proportional to $k T_{\mathrm{e}}$ (see Eq. (2.5)). Thus we claim that the model dependence of $L_{\mathrm{grb}} \propto\left(k T_{\mathrm{e}}\right)^{2}$ on the hot plasma temperature $T_{\mathrm{e}}$ explains the observed Amati relation in which $E_{\text {iso }}=\int L_{\mathrm{grb}}(t) d t \propto E_{\mathrm{p}}^{2}$. It is worth noting that the peak energy $E_{\text {peak }}$ of the emergent Wien spectrum should be equal to $3 k T_{\mathrm{e}}$ or $E_{\mathrm{p}}=E E_{\text {peak }}=4 k T_{e}$ for $E F(E)$-diagram.

An important prediction of our model is that the peak energy in the $\mathrm{EF}(\mathrm{E})$ diagram originates in the early sub-relativistic phase and is proportional to plasma temperature $k T_{\mathrm{e}}$ and the resulting luminosity $L_{\mathrm{grb}}$ is proportional to $\left(k T_{\mathrm{e}}\right)^{2}$. This dependence is the same, after cosmological corrections, in the source and observer frame. In fact, no fine tuning related to some Lorentz $\Gamma$-factor of relativistic expansion is required. We claim that this result of our model naturally explains the physical origin of the Amati relation.

\section{References}

Amati, L., Frontera, F., \& Guidorzi, C., 2009, A\&A, 508, 173

Amati, L., Guidorzi, C., Frontera, F., et al., 2008, MNRAS, 391, 577

Amati, L., Frontera, F., Tavani, M., et al., 2002, A\&A, 390, 81

Band, D., Matteson, J., Ford, L., et al., 1993, ApJ, 413, 281

Farinelli, R., Titarchuk, L., Paizis, A., \& Frontera, F., 2008, ApJ, 680, 602

Sunyaev, R.A., \& Titarchuk, L.G., 1980, A\&A, 86, 121 (ST80)

Sunyaev, R.A., \& Titarchuk, L.G., 1985, A\&A, 143, 374

Titarchuk, L., Farinelli, R., Frontera, F., \& Amati, L., 2012, ApJ, 752, 116 (TFFA12)

Titarchuk, L., Mastichiadis, A., \& Kylafis, N.D., 1997, ApJ, 487, 834 
